\title{
450 hidrotubaciones histero-tubáricas realizadas en el Centro Peruano de Fertilidad Matrimonial
}

\author{
Doctores Jorge Ascenzo-Cabello (*), Javier Hoyle C. (**), Rafael
} de la Puente L. (**), Vicente Chiong Ch., Roberto Ruíz G.

Todo especialista en esterilidad matrimonial siente cierta aprensión y justificada desconfianza en el éxito del tratamiento, cuando es el "Factor Tubario" el comprometido, en el diagnóstico cie las causas de la pareja estéril.

La experiencia ha demostrado que, los tratamientos médicos, con persuflaciones de anhídrido carbónico repetidas, ondas cortas, antibióticos parenterales $\mathrm{u}$ orales, los estrógenos y la histerosalpingografía diagnóstica, que a veces actúa también terapéuticamente, resuelven solo casos esporádicos. La plastía tubaria quirúrgica, con los métodos más modernos y aún seleccionando los casos, deja mucho que desear y pocos son los especialistas que se atreven a indicarla, asi como pocas las pacientes que permiten ser intervenidas después que se les refiere el reducido número de éxitos.

Los hechos anteriores nos han animado a probar otra clase đe tratamiento, básicamente médico: "la hidrotubación".

La hidrotubación hístero-tubaria consiste en inyectar una sustancia líquida, por el orificio externo del canal cervical, haciéndolo pasar a través de éste, de la cavidad uterina y luz tubaria, saliendo por el orificio peritoneal de los pabellones tubarios, hasta el peritoneo donde se reabsorberia.

A la acción benéfica que representa la presión de la ola hiaráulica sobre posibles adherencias flácidas intra-canaliculares,

(*) Secretario General Activo de la Asociación Internacional de Fertilidad.

(**) Sub-Director del Centro Peruano de Fertilidad Matrimonial.

(***) Secretario Nacional para el Perú de la Asociación Internacional de Fertilidad. 
rompiéndolas o desplazándolas, se agregaría la de diversos elementos añadidos a la sustancia líquida, con acción antibiótica y lítica. Además, al llegar al peritoneo, actuaría favorablemente sobre el "Factor Peritoneal" de esterilidad, que acompaña con tanta frecuencia al "Factor Tubario".

\section{METODO Y MATERIAL}

Hacemos rutinariamente hidrotubaciones en todo caso de factor tubario comprometido, sea o no el único factor causal de esterilidad. Para tener la certeza de un verdadero compromiso tubario, realizamos en ciclos distintos, dos persuflaciones con anhídrido carbónico, de preferencia la segunda con antiespasmódico parenteral previo y lo ratificamos o rectificamos por la histerosalpingografía, dados los numerosos casos de diagnóstico equivocado cuando se practica solo uno de los dos métodos anteriormente citados.

a) Número de hidrotubaciones y momento oportuno de practicarlas:

Las iniciamos alrededor del séptimo día de iniciada la regla, una cada tercero o cuarto día, en tres ciclos, con tres hidrotubaciones cada uno, total nueve. Indicamos en el cuarto ciclo nueva persuflación con anhídrido carbónico o histerosalpingografía para comprobar resultados.

\section{b) Precauciones:}

No haber metroanexitis activa actual; velocidad de sedimentación normal; no constatar ecto, ni endocervicitis, ni endometritis a la biopsia de endometrio reciente; no inyectar el líquido a demasiada presión, éste debe ingresar suavemente y el operador debe parar la presión sin ceder (sin hacer regresar el émbolo) cuando se nota hiperpresión endouterina y esperar el tiempo que sea necesario hasta que vuelva a ingresar fácilmente, si no ocurriera, no insistir con presión exagerada; dar rutinariamente antibióticos parenterales terminada la hidrotubación (Penicilina más estreptomicina de larga duración en una sola inyección).

\section{c) Técnica:}

Usamos la "Cánula Uterina de Kahn", previamente purgada con el líquido a inyectar, la adherimos al cervix con una pinza 
uterina de Schreöder, siguiendo igual técnica que al hacer una persuflación o histerosalpingografía.

Insertada la cánula, le adherimos una jeringa a su extremo libre, de 10 centímetros cúbicos, B. D., con pico de metal, e inyectamos la solución que para nosotros siempre contiene nueve centímetros cúbicos, generalmente al principio se produce una marcada resistencia que dura de treinta segundos a dos minutos y que cede expontáneamente. Lo importante es no vencerla con exceso de presión, sino manteniendo discreta, pero constante presión en el émbolo; cedida esta resistencia, inyectamos suavemente el resto, en dos o tres minutos, terminados de pasar los nueve centímetros cúbicos, cerramos la llave de paso del extremo libre y dejamos que la enfermera sostenga el instrumental; a los diez minutos regresamos y extraemos la cánula.

d) Composición del líquido inyectado:

Actualmente hacemos a cada paciente una receta que le alcanza para dos sesiones.

Rp.

Quemicetina Succinato "Erba" (Cloramfenicol succinato sal sódica, equivalente a 1 gramo de cloramfenicol levógiro sint. Un frasco. Wydase (hialuronidasa "Wyeth" 150 unidades F. E. U. (T. R.) aproximadamente 500 unidades viscosimétricas). Un frasco. Suero Fisiológico 20 centímetros cúbicos. Una ampolleta.

Ponemos 16 centímetros cúbicos de suero fisiológico en el frasco de Quemicetina Succinato y 2 centímetros cúbicos en el cie Wydase. Extraemos 8 centímetros cúbicos del primero y un centímetro cúbico del segundo, que hacen los 9 centímetros cú. bicos de nuestra solución que, por tal, tiene: $1 / 2$ gramo de cio ramfenicol levógiro sint., mas 75 unidades F. E. U. (T. R.) o 250 unidades viscosimétricas de hialuronidasa.

c) Experiencia con otras sustancias:

Probamos al principio la Omnamicina "Hoechst"; mezcla de penicilina, estreptomicina y una vacuna inespecífica la omnacina; que inyectamos cinco minutos después de introducir la hialuronidasa y aunque obtuvimos resultados aceptables y algunas gestaciones, la suprimimos por sentir las pacientes diversas molestias al hipograstrio, post-hidrotubaciones.

La eritromicina (illoticina) produjo ardor hipogástrico desproporcionado; el clorhidrato de tetraciclina (tetracyna) aunque 
menos que la oxitetraciclina (terramicina), produjo como ésta, ardor, tensión y dolor hipogástricos post-hidrotubación, a veces, inmediatamente y otras tardíamente, más o menos prolongados que nos obligó a suspenderlas.

Más de la mitad de nuestras primeras hidrotubaciones (56.8\%) las practicamos con "Ambistryn" Squibb: sulfatos de dihidroestreptomicina y estreptomicina: un gramo que mezclamos con la misma cantidad de Wydase, también en nueve centímetros cúbicos de suero fisiológico; esta solución nos dio resultados discretamente inferiores que con el cloramfenicol (succinato de quemicetina) y aunque casi no produjo dolor ni ardor, no se pudo comparar con la falta de molestias y gran aceptación de las pacientes, al succinato de quemicetina; además, las dos únicas reacciones peritoneales que tuvimos, una de ellas muy discreta y el único shock que se observó, también discreto, ocurrieron con el ambistryn. Sin embargo, las siete gestaciones que tuvimos se produjeron cuando intervino la estreptomicina: ya sea unida a la penicilina, como fue con la omnamicina, o en forma de ambistryn, ya puro o intercalado con la quemicetina o terramicina.

\section{f) Tratamiento de refuerzo:}

Al $50 \%$ de las hidroturbaciones se les añadió 20 aplicaciones de ondas cortas al hipogastrio con anterioridad, simultáneamente o posteriormente a la hidrotubación.

Hemos agregado también, en algo menos de la cuarta parte de los casos, $(21.0 \%)$, prednisona por vía oral en dosis que fluctuaban entre los 15 y 20 miligramos diarios durante 60 días (Decortancyl, "Rousel" comprimidos de 5 miligramos).

\section{RESULTADOS}

Hemos practicado 450 hidrotubaciones en 81 mujeres, que da un promedio de $5.5 \%$ hidrotubaciones por paciente. De éstas fueron estériles primarias el $71.0 \%$. Partimos de 43 pacientes con trompas ratificadamente obstruídas: $53.0 \%$ y 38 con trompas comprobadamente estenosadas: $46.9 \%$.

De estas 81 pacientes en 26 aún no tenemos los resultados por estar en tratamiento, 6 han abandonado y en 2 lo hemos suspendido: en un caso por sensibilidad marcada a los antbióticos y en otro por haber fracasado reinteradamente a los antibióción anatómica manifiesta. Esto nos totaliza, 34 pacientes que 
no han tenido comprobación de resultados en el cuarto ciclo, como es nuestra costumbre.

De las 47 pacientes restantes, obtuvimos los siguientes resultados:

7 gestaciones: $14.8 \%$.

15 pacientes con factor tubario normalizado: $31.9 \%$.

13 pacientes con factor tubario mejorado: $27.6 \%$.

12 fracasos: $25.5 \%$.

Uniendo las pacientes que han obtenido gestaciones con las de factores tubarios normalizados y mejorados, observamos un número global de $74.3 \%$ de buenos resultados.

Las ondas cortas, dadas al mismo tiempo que las hidrotubaciones, mejoran el efecto de éstas en un $20 \%$, el resultado ha sido más favorable cuando se aplicaron simultáneamente con ellas, (el día que la mujer hace la hidrotubación, no hace las ondas cortas).

Con la prednisona (Decortancyl "Roussel"), administrada en la forma ya citada, hemos observado positiva mejoría en los resultados de estas hidrotubaciones que vieron mejorar sus efectos benéficos en un $30 \%$. Como hecho notorio, y que recalcamos, el $83.3 \%$ de las mujeres que embarazaron con las hidrotubaciones habían recibido simultáneamente prednisona (Decortancyl), en un tercio de estos casos, se administró la prednisona simultáneamente a las hidrotubaciones, en el otro anteriormente a ellas y en el último tercio posteriormente, sin haber observado diferencias ostensibles en sus beneficios.

Como complicaciones hemos tenido: dos casos de pelviperitonitis localizadas, una de ellas discreta y ambas resueltas médicamente y un cuadro de shock no severo, que hace un total de $2.7 \%$ de complicaciones.

\section{DISCUSION}

Cuando el "factor tubario" está comprometido con certeza en el matrimonio sin hijos, no escapa a ningún especializado observador los gruesos antecedentes que acompañan a la inmensa mayoría de estos casos; en efecto, hemos encontrado, que:

21 pacientes $(27.6 \%)$, habían sido previamente operadas: 9 sobre trompas (3 plastias tubarias sin resultados, 3 salpingectomías, 2 embarazos ectópicos y un hematosalpings).

3 habian sido intervenidas sobre ovarios (2 por quiste del ovario y una por tubercoloma del ovario). 
4 operadas sobre el útero (3 miemectomías, una por retroflexión).

5 con afecciones varias (4 apendicectomías, 2 supuradas, y una por endometriosis).

Ya sabemos que las intervenciones quirúrgicas dejan generalmente adherencias y condiciones poco apropiadas para la migración del úvulo y cuando no dan resultados favorable, después de un tiempo de espera prudencial, son casos pésimos para cualquier otro tipo de tratamiento.

Por otra parte, habían 15 pacientes con metroanexitis manifiesta previa, que fue necesario tratar, y 2 con hidrosalpings.

10 casos tenían antecedentes de 2 o más abortos, siendo la mayoría criminales y un tercio de ellos con reacción febril o inflamación pelviana post-curetaje, también 2 casos de infección puerperal. Grueso antecedente de tuberculosis en 11 mujeres, en un tercio de las cuales había localización genital.

La observación serena del $74.3 \%$ de buenos resultados, o sea ia suma de pacientes que han gestado y que han normalizado 0 mejorado francamente el "Factor Tubario" comprometido, obte. nidos con las hidrotubaciones, nos lleva a aconsejarlas, dado el alto porcentaje de éxitos, a pesar de los gruesos antecedentes que las hacían malas candidatas para resolver su problema, con cualquier tipo de tratamiento.

Nos llama la atención el hecho que hasta la fecha no se ha producido ningún embarazo ectópico, cosa que desde luego es lógico que pueda ocurrir por razones obvias. Incluso, tenemos el caso, de una señora que hizo años atrás una pelviperitonitis tuberculosa, con ascitis y porsteriormente un embarazo ectópico; sin ninguna expectativa de éxito y pensando en la gruesa posibilidad de otro embarazo ectópico, nos arriesgamos a abrir la otra trompa con hidrotubaciones con ambistryn, habiendo obtenido un embarazo perfectamente uterino (Historia número 564). En conclusión, pues, tenemos $0.0 \%$ de embarazos ectópicos hasta el momento.

Creemos que con el succinato de quemicetina, todavía no hemos obtenido gestaciones, debido a que lo venimos utilizando rutinariamente desde hace relativo poco tiempo y los resultados en cste sentido deben ser esperados; en cambio, la apertura de las trompas ha sido ya constatada en la mayoría de los casos. Esperamos impacientemente estos resultados, en mérito a que el $42.8 \%$ de las gestaciones las hemos obtenido con "Wydase" y om- 
namicina, sustancia que ya hemos abandonado por razones que comentamos anteriormente y el $57.1 \%$ con "Wydase" y ambistryn alternados con quemicetina, terramicina o solos.

\section{RESUMEN Y CONCLUSIONES}

19 Practicamos hidrotubaqiones histero-tubáricas en todos los casos de "Factor Tubario" comprometido con certeza, exista o no otro factor de esterilidad matrimonial.

2 Indicamos 9 hidrotubaciones, 3 por ciclo, durante 3 meses, al 4 c ciclo comprobamos resultados.

3o Describimos la técnica de las hidrotubaciones y sus contraindicaciones.

4 o Preferimos actualmente, que la solución inyectada contenga $1 / 2$ gramo de succinato de cloramfenicol levógiro sintético, (quemicetina succinato "Erba"), más 250 unidades viscosimétricas de hialuronidasa (Wydase 150 unidades F. E. U. (T. R.) "Wyeth"), diluídas en 9 centímetros cúbicos de suero fisiológico.

5 o Explicamos por qué hemos abandonado el ambistryn, omnamicina, terramicina y tetracyna y la experiencia obtenida con ellos.

6 ${ }^{\circ}$ Hemos practicado 450 hidrotubaciones en 81 mujeres, con un promedio de $5.5 \%$ hidrotubaciones por paciente, las que fueron estériles primarias en un $71.0 \%$.

7 Iniciamos el tratamiento con trompas ratificadamente obstruídas en el $53.0 \%$ de los casos y con trompas comprobadamente estenosadas en el $46.9 \%$.

8 o De las 47 pacientes en las que se pudo comprobar los resultados obtenidos, se halló, lo siguiente:

$14.8 \%$ de gestaciones, $34.9 \%$ de factor tubario normalizado,

$27.6 \%$ de factor tubario mejorado y $25.5 \%$ de fracasos.

9? Uniendo las gestaciones con los "Factores Tubarios" normalizados y mejorados, obtenemos un $74.3 \%$ de buenos resultados.

10. Ocurrieron complicaciones en el $2.7 \%$ de los casos; todas pudieron resolverse en forma feliz, con tratamiento médico.

11. Describimos detalladamente los numerosos antecedentes quirúrgicos y de otro tipo, que hacían malos estos casos para desobstruir sus trompas y lograr hijos, por lo que indirectamente podemos concluir, que los resultados habrían sido mejor si hubiéramos deseado seleccionar las pacientes. 
12. En el $56.0 \%$ de los casos administramos ondas cortas simultáneamente y hemos observado que mejoran los resultados de las hidrotubaciones en un $20 \%$.

13. La prednisona que suministramos en el $21.0 \%$ de estas mujeres y cuya técnica de administración describimos, mejoró los beneficios de las hidrotubaciones en un $30 \%$.

14. En el $42.8 \%$ de los casos en que se obtuvo gestación, intervino la omnamicina, en el $57.1 \%$ el ambistryn, que en $1 / 3$ se combinó a la terramicina, en el otro unido a la quemicetina $y$ en $1 / 3$ solo.

15. Hacemos ver que no hemos obtenido aún gestaciones en casos en que se usó la quemicetina porque su aplicación es reciente, pero en cuanto a la mejoría del "Factor Tubario" y a la falta completa de molestias, es discretamente superior al ambistryn.

16. En el $83.3 \%$ de los casos que gestaron, se administró también prednisona, no encontrándose diferencia si la administración fue antes, durante o después de las hidrotubaciones.

17. La estadística nos parece corta para sacar conclusiones clefinitivas, pero a nuestro concepto las hidrotubaciones, mejor aún, combinadas con ondas cortas y prednisona por vía oral, abren un nuevo jalón, con resultados positivamente benéficos en el arsenal terapéutico contra el "Factor Tubario" comprometido, que hasta ahora había obtenido resultados aleatorios.

\section{SUMMARY AND CONCLUSIONS}

1. We perform hystero-tubal hydrotubations, in all cases where the "Tubal Factor" is involved with absolute certainty.

2. We indicate 8 hydrotubations, 3 per cycle, during 3 months, in the 4 th cycle we verify results.

3. We describe the technique of the hydrotubations and their contraindications.

4. At present we prefer the injected solution to contain $1 / 2$ gm. of "Erba" lavoratory synthetical chloranphenicol succinate ("Erba" Kemicetine Succinate) plus 250 visc. units of hyaluronidase (Wydase 150 F. E. U. (T.R.) "Wyeth" units) diluted in 9 c. c. of isotonic saline solution.

5. We explain the reason why we have discontinued the use of ambistryn, omnamicyn and tetracyn and the experience obtained with same. 
6. We have performed 450 hydrotubations, in 81 women, an average of 5.5 hydrotubations per patient of which $71.0 \%$ was primary sterile.

7. Initiated the treatment with obstructed tubes in $53.0 \%$ of the cases and with stenosied tubes in $46.0 \%$.

8. Our findings, in 47 patients in which results could be verified, were the following:

$14.8 \%$ pregnancies, $34.9 \%$ of normalized tubal factor. $27.6 \%$ improved tubal factor and $25.5 \%$ of failures.

9. The sum of pregnancies plus normalized and improved. "Tubal Factors" give a total of $74.3 \%$ of good results.

10. There were two complications in the $2.7 \%$ of the cases, all were satisfactorily solved by medical treatment.

11. We give a detailed description of the numerous sugical antecedents and other antecedents which made these cases difficult for the desobstruction of the tubes for having children; from this we indirectly conclude that the results would have been better if we would have wanted to select the patients.

12. In $56.0 \%$ of the cases we give Short Waves simultaneously and we have observed thah hydrotubations results improve in $20 \%$.

13. Prednisone administered in $21.0 \%$ of these women and which administering technique we describe, improved the benefits of the hydrotubations in $30 \%$.

14. In $43.8 \%$ of the cases in which a pregnancy was obtained, omnamicyn intervened, in $57.1 \%$ ambistryn which in $1 / 3$ was combined with terramycin, in the other third mixed with Kemicetine and in $1 / 3$ alone.

15. We wish to point out that we have not obtained pregnancies with kemicetine as yet, due to the fact that its administration is recent, but regarding the improvement of the "Tubal Factor" and to the complete absence of discomfort it is somewhat superior to the ambistryn.

16. In $83.3 \%$ of pregnancies, prednisone was administered, we found no difference when the administration was before, during or after the hydrotubations.

17. These statistics are rather too brief to enable us to arribe to definitive conclusions, but to our knowledge, the hydrotubations, even better combined with Short Waves and Prednisone oral administration, open a new progress with positive beneficial results in the therapeutic arsenal against the "Tubal Factor" involved, which up till now had obtained aleatory results. 


\section{BIBLIOGRAFIA CONGULTADA}

BRUNO ROQUE OSCAR.-Adaptación del salpingoquimógrafo para la práctica de la hidrotubación. Obs. y Gin Lat.-Amer. XI:155, 1953.

DO AMARAL FERREIRA CLARICE y ALDEIA VIRGILIO B.--Hidrointubacao medicamentosa no tratamento das obstrucçoes tubarias. Anais Bras. de Gin. 43:185, 1957.

DRAGULESCU C. PAUL.-Exploración Semiológica de la Permeabilidad de las Trompas de Falopio por Hidrotubación. Anales de la Univ. Cent. de Venezuela. XLI-Julio, 1956.

ELIAS COSTA JORGE D. y GUGLIELMONE PEDRO.-Estrepcinasaestrepto-dornasa en hidrotubaciones en el tratamiento de la esterilidad por obstrucción tubaria. Obst. y Gin. Lat.-Amer. XIV:21, 1956.

MURRAY EDMUNDO G. y FOIX ANTONIO.-Hidrotubaciones con hialuronidasa y antibióticos en el tratamiento del factor tubárico. Obst. y Gin. Lat.-Amer. XV:84, 1957.

RUEDA-GONZALEZ RICARDO.-Valoración de los métodos de exploración de la función tubaria. Rev. Colombiana de Obst. y Gin. VIII:115, 1957.

VASPASIANO RAMOS A. y PEANO MARCEL.-Tratamiento médico dos obstrucoes tubarias. Anais Bras. de Gin. 41:389, 1956.

YAGI HIDEO.- Eeparate Examination of the Patency of Each Tube with Isotonic Saline Solution. Fertil. \& Steril. 6:550, 1955. 